\title{
UNE THÉORIE DES FICTIONS PEUT-ELLE ÊTRE UTILE EN ÉPISTÉMOLOGIE? Jean-Pierre Cléro
}

Christian Laval, Jeremy Bentham. Le pouvoir des fictions. Paris, Presses universitaires de France, 1994. $18 \times$ 11,5, 128 p. (Philosophies).

La parution d'un livre sur Jeremy Bentham, ne comptât-il que cent vingt-cinq pages, reste un événement, tant la philosophie de cet auteur est encore mal connue, en France notamment. On sait que Bentham est le père peu fréquentable d'une philosophie morale réfutée dans tous les bons manuels scolaires; on sait, grâce à Michel Foucault, qu'il est l'auteur d'un projet de «panoptique »; on ne sait généralement pas encore qu'il est l'auteur d'une théorie originale des fictions. Or c'est sous cet angle heureusement choisi que Christian Laval développe les thèses de Bentham, comme étant celles d'une philosophie fondamentale dont notre temps pourrait encore tirer profit dans un projet de mise en ordre de multiples formes culturelles : politiques, juridiques et morales, bien sûr ; économiques, évidemment; mais aussi scientifiques, qu'il s'agisse des sciences dites « humaines » ou des autres.

L'immense mérite du livre de Laval réside moins dans le problème qu'il est censé traiter ${ }^{1}$ - lequel, en réalité, n'unifie pas l'ouvrage - que dans deux positions essentielles et fermement tenues. La première consiste dans le pari de faire connaître à un large public des textes mal connus, voire ignorés, peu trouvables d'ailleurs dans les bibliothèques françaises, extraits des Fragments on ontology et de Chrestomathia, textes présentés fragmentairement par Charles Kay Ogden ${ }^{2}$ et par Biruk Parekh ${ }^{3}$, et dont une

1. L'auteur, p. 6, avoue s'être intéressé, « dans l'utilitarisme », à « la jointure nécessaire de la revendication individualiste du moi et de la mise à nu des déterminations symboliques du sujet ». Le problème était déjà celui de Hume, qui avait critiqué la substantialité du moi, la faisant éclater en une multitude de fictions qui ne retrouvent une unité illusoire et toujours provisoire que dans le jeu des passions. Chez Bentham, si le schème est globalement le même, le détail est évidemment différent, puisque la dynamique passionnelle n'est plus qu'un cas particulier d'une dynamique fictionnelle, plus générale.

2. Charles Kay OGDEN, Bentham's theory of fictions, Londres, 1932.

3. Biruk PAREkH, Bentham's political thought, Londres, Croom Helm, 1973.

Revue de synthese : $4^{\mathrm{e}} \mathrm{S}$. $\mathrm{n}^{\circ} 4$, oct.-déc. 1998, p. 613-631. 
traduction générale manque encore cruellement dans notre langue. La seconde tient dans la volonté affirmée et parfaitement justifiée de l'auteur de ne jamais désolidariser l'utilitarisme d'une philosophie des fictions. Les clichés ordinaires sur la philosophie de Bentham ont complètement caché ce point : on n'a pas compris que le jeu complexe des représentations s'articulait sur une dynamique de l'utile, qui fait apparaître ces représentations comme autant de fictions, mais qui ne peut elle-même, si elle est conséquente (comme elle l'est chez le père de l'utilitarisme), s'exprimer qu'à travers des fictions. Car si Bentham dénonce, au nom de l'utilité, un certain nombre de fictions, il n'omet pas de dire que l'utilité aussi est une fiction.

Nous sommes dès lors au coeur des difficultés de la philosophie utilitaire : si l'utilité fait apparaître presque tous les concepts comme des fictions plus ou moins fallacieuses, pourquoi, s'estimant elle-même comme une fiction, devrait-elle s'estimer mieux fondée que les autres? Qu'est-ce qui donne le droit à celui qui fait apparaître les concepts comme des fictions de distinguer des fictions bien fondées de fictions qui ne le seraient pas («fallacies »)? Comment peut-on corriger des fictions par d'autres? Si Bentham a attaqué le scepticisme de Hume, est-il tellement sûr qu'il nous en sorte?

Ce problème, inévitable, est à plusieurs reprises posé par Laval sous des aspects différents ${ }^{4}$, mais - et c'est peut-être la limite d'un ouvrage qui, s'il intéressera les philosophes, s'il s'inscrit dans une collection de «Philosophies ", n'est pourtant pas lui-même un ouvrage de philosophie - il ne parvient pas à tendre, d'un bout à l'autre, l'ouvrage. Ainsi l'auteur croit-il voir des solutions dans des problèmes qui ne font que rebondir.

Ainsi est-il frappant de remarquer qu'après avoir posé la question de savoir comment on peut, par des fictions, connaître qu'il existe des fictions, et comment certaines d'entre elles peuvent apparaître mieux fondées que d'autres (par exemple, sous l'angle de la justice), l'auteur croit pouvoir nous assurer qu'une analyse du langage permettra de nous sortir d'affaire : "Toute réforme politique devra passer par une maitrise de la langue, qui supposera elle-même l'établissement d'une science fondamentale du langage $^{5}$. " Sans doute Bentham montre-t-il mieux que personne comment le langage se stratifie en de multiples niveaux de lui-même par lesquels il s'éloigne du degré de l'expérience sensible; mais si l'on comprend bien par

4. Par ex., l'auteur demande, p. 81, si « cette théorie du langage et des fictions est compatible avec la connaissance scientifique. Celle-ci concerne le projet de reconstruction utilitariste à plus d'un titre, et d'abord en ce qu'il prend appui sur l'objectivation scientifique de la conduite humaine. Si la science est un songe, la légitimité du projet ne tient plus ». Et, un peu plus loin, p. 88, la question rebondit : «Comment [la connaissance] est-elle possible si elle doit obligatoirement passer dans le registre de la fiction? "

5. P. 37. Cet optimisme langagier se voit aussi p. 38,81 . 
là comment le problème se pose, on ne le résout pas et un problème déplacé n'est pas pour autant réglé. Bentham, d'ailleurs, n'y songe guère, qui, s'il privilégie le langage dans son étude des fictions, ne prétend pas que toutes les fictions sont langagières et fait du langage même un produit des fictions ${ }^{6}$. S'il fallait trouver une esquisse de solution à ce problème, ce serait plutôt du côté du délicat jeu des négations qui apparente, de ce point de vue, la philosophie de Bentham à celle de Pascal $^{7}$ : quand la vérité ne peut être saisie directement, elle peut encore l'être par des voies apagogiques. Peut-être le meilleur service à rendre aux philosophes serait-il moins de faire la doxographie d'une théorie du langage - fût-elle intéressante - que de suivre le subtil jeu des négations ${ }^{8}$. La vérité n'est pas un dogme; Laval le sait bien, mais ne lui aurait-il pas fallu éviter de présenter une théorie du langage comme si elle pouvait en être un?

D'autant que cette présentation du langage comme « solution " dissimule les meilleurs apports de Bentham, ceux qui ne mettent pas en avant les critères de clarté du langage. Un langage clair n'est pas nécessairement un langage vrai : les «fallacies " pèchent-elles toujours par défaut de clarté ? Seraient-elles si convaincantes si elles n'étaient souvent si claires? Pas plus chez Bentham que chez Edmund Burke, la clarté n'est un critère de vérité. Comment le jeu complexe des démultiplications du langage pour faire apparaître ses propres fictions pourrait-il, sinon par miracle auquel personne ne croit, devenir clair? Dès lors, comment être tout à fait convaincu par la recette qui consiste à faire passer la conception benthamienne de la loi «par l'établissement de classifications et de dénominations claires et pratiques qui permettent d'atteindre chaque sujet le plus facilement $"(\text { p. 38, 45, 46, etc. })^{9}$. Y aurait-il donc une double philosophie du langage chez Bentham, exotérique et ésotérique, comme il y aurait (ainsi que nous le suggérerons) une double approche de la raison?

Si l'exemple du langage est privilégié ici, c'est parce que Laval luimême lui consacre la majeure partie de son ouvrage; mais il serait possible de se livrer à l'analyse des mêmes insuffisances sur des notions aussi

6. Si Bentham dit un peu imprudemment que « la fiction est une espèce de réalité verbale ", c'est pour ajouter presque aussitôt qu'elle est tout autant « la condition sans laquelle la matière du langage n'aurait pu se constituer , voir op. cit. supra n. 2, p. 137. Toutefois, il manque peut-être chez Bentham, comme le suggère Christian Laval, p. 84, une analyse du rapport de la fiction et de l'imagination.

7. Ce n'est pas d'ailleurs le seul point commun important de Bentham et de Pascal. On trouve, chez l'un et chez l'autre, le même abrupt de certaines formules sur le langage. Si le langage peut être considéré « comme morale et législation » (p. 62 sqq.) chez Bentham, il est science chez Pascal (Pensées, éd. Léon Brunschvico, fragm. 912).

8. Laval a bien vu d'ailleurs ce sens de la négation lorsqu'il parle des lois, voir p. 12, 23 : « La loi est fondamentalement interdictrice. " $1 \mathrm{l}$ aurait pu le voir à l'œuvre sur tous les autres registres.

9. Il semble que Laval lui-même, p. 51, ait opposé et subordonné, en une belle formule « le tout voir du panoptisme benthamien au tout dire de la logique de la volonté ». 
importantes que l'autorité ou la raison. Laval laisse en quelque sorte celles-ci en fuite, sans montrer suffisamment les enjeux dont elles sont l'objet. Si l'on peut découvrir l'autorité comme «fallacy» (par ex., p. 34), elle prend aussi un sens dans une théorie qui généralise les fictions et montre comment elle est l'artisan d'une transcendance (c'est-à-dire d'une position d'objets comme s'ils avaient une réalité); dès lors, il n'est plus question de la rejeter, car l'utilité même se définit comme autorité ${ }^{10}$ et ce que nous appelons le vrai ne peut guère procéder que d'un jeu des autorités entre elles. Et sans doute est-ce lui, peut-être de façon aporétique, qu'il fallait commencer à explorer.

De même, on a le sentiment, en lisant le texte de Laval, que l'utilitarisme est un rationalisme classique qui découvrirait des «fallacies » du point de vue d'une raison sûre d'elle-même et de son bon droit, comme si la leçon de Hume - auteur fort critiqué sur d'autres points par Bentham ${ }^{11}$ n'avait pas porté de façon décisive : la raison est une construction fictive et c'est par cette construction fictive qu'il faut promouvoir et dénoncer des fictions. Mais comment cela se peut-il ? On peut bien dénoncer la fausseté de ceux qui attaquent toute innovation du point de vue d'une prétendue sagesse des Anciens, mais il ne s'agit pas non plus de dire qu'une innovation, parce qu'elle est innovation, est mieux fondée; dès lors, on n'échappe pas à la question du bon fondement et il faut, pour la traiter, autre chose qu'une position dogmatique, laquelle, à coup sûr, ne convaincra pas le philosophe.

La question est même tellement aiguë chez Bentham qu'elle est beaucoup plus délicate à résoudre que chez Hume. Par son sentimentalisme, Hume compensait une décomposition critique dangereuse sur le plan pratique; et, sur le plan théorique, il faisait jouer à un certain vitalisme la même fonction. Bentham n'a même plus ce recours dès lors qu'il attaque le

10. Cité par Laval, p. 32 : « Bentham a justement voulu faire de l'utilité cette "autorité qui corrige le vice originel de la structure du langage". " L'auteur du Pouvoir des fictions se montre très sensible, par plusieurs détails, à cette philosophie de l'autorité chez Bentham. Ainsi remarque-t-il, p. 41, dans l'expression de la volonté, outre « une précision dans la fin recherchée [...] la résonance d'une menace ou d'une récompense et la prise en compte dans la formulation des positions hiérarchiques et donc des rapports de force ». Il parle, à la page suivante (p. 42), des « pistes » ouvertes par Bentham lorsqu'il lie « la parole à la contrainte, en montrant la force de commandement du langage, et en articulant la grammaire à la jouissance ". Laval, p. 42, 57, rattache finement l'autorité à une philosophie du corps que Bentham hérite des Observations on man de David Hartuey. Il voit, par ailleurs, très bien le rattachement de Bentham à Hobbes sur ce thème de l'autorité (p. 46). On trouve aussi de belles formules qui synthétisent le propos, comme p. 75 : « Gouverner par les sensations impose de maîtriser la langue. Ailleurs, p. 85, les entités fictives sont heureusement appelées des « obligations langagières ", avec un jeu subtil sur les diverses significations du mot « obligation ".

11. En part., sur la question du sentimentalisme en morale. Plus généralement, le « pathémique ", loin d'être, comme c'est le cas chez Hume, le fondement du « symbolique ", est fondé par celui-ci et n'en est, tout au plus, qu'une partie. 
sentimentalisme humien et y reconnaît une gerbe de «fallacies ». Ce n'est toutefois pas une raison pour faire de Bentham un rationaliste. En ce sens, le statut du rationalisme chez Bentham - question incontestablement difficile et, sans doute, non résolue - nous semble malencontreusement rapprochée par l'auteur de celui qui existe chez Kant. Les relations dont parle Bentham ne sont pas des catégories a priori de l'entendement et la science ne consiste pas en l'application de catégories. La méthode benthamienne pour s'avancer dans un travail critique quelconque est beaucoup plus empiriste. Il s'agit tout autant de forger des abstractions que de s'en méfier, en n'oubliant jamais qu'elles ne sont que des abstractions dont les délimitations ne correspondent à rien de réel, quoiqu'elles permettent de travailler. À la différence de ce qui se passe chez Kant, la structure a priori de l'entendement ne peut être qu'un fantasme projeté à l'orée de ce travail, sans aucune réalité.

Ne conviendrait-il pas de distinguer deux groupes de textes chez Bentham - sinon une double philosophie, ce qui équivaudrait à une hypocrisie? D'une part, ceux qui, pour procéder rapidement à une attaque, se donnent les facilités d'un rationalisme (ce qui est à peu près le point de vue du Handbook of political fallacies); d'autre part, ceux qui, plus subtils et plus philosophiques, tiennent compte de la leçon humienne et tâchent de sortir, avec plus de difficultés que Hume lui-même, de la redoutable situation issue d'une critique du rationalisme. La genèse de la raison est insuffisamment prise en compte par l'auteur, qui finit par rattacher Bentham à une tradition rationaliste et à le désolidariser du courant empiriste, après avoir promis (p. 7) de voir en Bentham « l'authentique héritier des philosophies anglaises et françaises ». En tout cas, il semble bien difficile de souscrire au jugement de Laval, lorsqu'il nous dit que « Bentham a essayé [...] avec bien moins d'ampleur que Kant, de résoudre les difficultés ${ }^{12}$ nées de l'inadéquation de l'empirisme aux développements de la science " (p. 94).

Pourtant, les questions que nous venons de poser concernant la possibilité de la connaissance, l'autorité, le rationalisme ne sont pas oiseuses et inutiles de telle sorte qu'elles n'intéresseraient que le seul philosophe, soucieux de projeter ses propres problèmes sur les cuvres qu'il lit et les questions qu'il touche. L'utilitarisme ne peut intéresser personne tant qu'il ne se

12. Lesquelles? Et pourquoi seraient-elles mieux résolues par le kantisme dont la table des catégories n'a jamais représenté qu'une étape périmée de la science et dont la philosophie du langage brille par son absence - ce qui n'est évidemment pas le cas dans la tradition empiriste?

Dans le même ordre de critiques, et pendant que nous réglons quelques détails, il est difficile de lire sans sursauter, p. 40, que « la logique depuis Aristote ne s'est intéressée qu'au rapport du langage à la faculté de l'entendement. Elle a entièrement manqué le rapport du langage à la volonté ». Sans doute la Rhétorique ne s'identifie-t-elle pas à une logique, mais on trouve de nombreux éléments d'une logique de la volonté à travers son livre II sur les passions, par ex. 
justifie qu'à la manière d'un dogme. On fait œuvre utile lorsqu'on montre pourquoi l'utilitarisme est l'indication d'un chemin à prendre pour, sinon sortir des fictions (ce qui n'est pas possible), du moins s'orienter en elles. Bentham a manifestement cherché à découvrir, dans un jeu de forces, dont il a jeté à la fois les fondements et les premières esquisses du calcul, un dépassement du système des représentations; et peut-être a-t-il cru que l'on pouvait agir plus directement et plus efficacement sur les forces que sur les représentations, et que la représentation suivrait dès lors que la répartition du plaisir et de la douleur, selon le fameux principe de l'utilitarisme, était satisfaite. Il se peut d'ailleurs que l'utilitarisme ne rencontre qu'illusion dans cette direction, les premiers succès dans la recherche de l'organisation des forces nous faisant croire qu'elles pourraient jouir, à l'égard des représentations, d'une autonomie à laquelle, en réalité, elles ne sauraient atteindre ${ }^{13}$.

Ne pourrions-nous pas ici faire l'hypothèse d'une vérité du point de vue des forces par rapport à une plus grande versatilité et relativité des représentations? Il semble que Laval ait perçu quelque chose de ce processus lorsqu'il souligne l'intérêt tout particulier des réflexions sur la douleur chez Bentham. Il y a une valeur ontologique de la douleur (p. 83); peut-être la douleur est-elle, avec le plaisir, et sans doute plus que lui, le sérieux d'un jeu de fictions et le sérieux du passage d'un jeu de fictions à un autre.

Peut-être aussi Bentham a-t-il vu une "solution " du problème sceptique, par laquelle il entend se démarquer de Hume, en "historicisant " les fictions; ce qui nous a paru être, sinon un des thèmes du livre de Laval, du moins un élément fondamental de sa charpente, puisque, après l'analyse d'anciennes fictions, l'ouvrage s'achève longuement sur « l'ère des nouvelles fictions ». Contrairement à la légende, forgée par Élie Halévy, d'un Bentham méprisant l'histoire ${ }^{14}$, il se pourrait bien que Bentham ait vu la clé du problème «sceptique " (des fictions qui valent mieux que d'autres) dans un dépassement historique des unes par les autres. Mais ne voyons pas trop vite ici une «solution », car tout déplacement de fictions, qu'il s'inscrive dans une dynamique ou dans la temporalité plus ou moins longue de l'histoire, n'effectue pas nécessairement un bon travail : il y a des déplacements qui ne changent rien, d'autres qui déguisent, etc. Il resterait, de plus,

13. Les difficultés de ce genre de philosophie n'apparaissent pas dans la phase « critique ». Tant qu'on en reste à détecter le jeu des forces derrière les représentations, à voir comment un jeu de représentations se transforme dans un autre, il n'y a pas de difficultés. En revanche, lorsqu'on s'est suffisamment avancé pour comprendre le fonctionnement du jeu des forces et qu'on croit pouvoir le faire fonctionner de façon quasi autonome, les difficultés deviennent considérables.

14. Élie Halévy, La Formation du radicalisme philosophique, 3 vol., Paris, 1901-1904, vol. III : Le Radicalisme philosophique, p. 453, n. 12: « Bentham méprise l'histoire aussi absolument que pouvait faire un cartésien. » Les arguments produits par Halévy pour le montrer sont ou d'une extraordinaire futilité ou de mauvaise foi. 
à connaître par quels liens la dynamique se rattache à cette nouvelle histoire dont Bentham a esquissé quelques traits en deux articles fondamentaux ${ }^{15}$.

Le texte de Laval, dans sa sinuosité et sa complexité, par le choix d'une réflexion sur Bentham, nous semble poser le problème essentiel d'une philosophie qui pourrait être utile aux sciences de notre temps et, particulièrement, à la linguistique, à la sociologie, à la psychologie, à la psychanalyse surtout. Laval cite toujours judicieusement des phrases de Jacques Lacan (p. $5,25, \ldots)$ qui montrent l'intérêt que l'auteur des Écrits, du VII livre du Séminaire, d'Encore, pouvait prendre à ce thème des fictions chez Bentham; peut-être envisageait-il de ce côté une théorie générale de la culture $^{16}$. Mais il faut, pour qu'elle puisse être utile, se détourner plus nettement des voies du dogmatisme et de la doxographie. D'abord, il faut renoncer à l'idée que les sciences humaines puissent terminer une réponse à des questions philosophiques; sans doute, posent-elles des questions philosophiques, sans doute exigent-elles du philosophe qu'il affine ses questions et les exigences de ses réponses. Mais ce serait vraiment une erreur de penser qu'il faille attendre d'une recherche linguistique, par exemple, une réponse à la question philosophique des valeurs différentes de divers types de fictions. En second lieu, ce n'est pas parce que la philosophie pose des questions auxquelles ni elle ni les sciences humaines ne répondent, qu'elle en est moins utile à ces dernières. Sans doute, la philosophie peut-elle essayer d'offrir une structure générale de mise en ordre des sciences de son temps; Bentham en a incontestablement rêvé et il est même passé à l'acte, en continuateur critique du projet encyclopédique, dans Chrestomathia. Mais ce n'est certainement pas seulement par cette structure que la philosophie intéresse les sciences humaines : si elle leur est utile, c'est sans doute par la permanence aporétique de l'ouverture de ses questions au fondement et à l'orée de tout problème scientifique. Il faut sans doute que, pour poser adéquatement ses questions, le philosophe soit informé des méthodes et des résultats des sciences humaines; mais il serait d'une incroyable maladresse que le spécialiste de quelque science humaine s'avance sur le terrain de la philosophie pour prétendre refermer une question aussi fondamentale que la distinction de divers types de fictions. La philosophie est utile aux sciences, tant qu'elle ne se présente pas comme un dogme; les sciences - et tout particulièrement les sciences humaines sont utiles à la philosophie tant qu'elles ne se présentent pas trop facilement comme des solutions aux problèmes qu'elle repère.

15. Voir, in Jeremy Bentham, Deontology, éd. Ammon Goldworth, Oxford, Clarendon Press, 1983, l'Article on utilitarism, longue et brève version, p. 289 sqq.

16. Laval, p. 7, parle plutôt d'une « théorie du symbolique ». 
Le problème qui se pose, par-delà ce livre de Laval sur Bentham, est de savoir si une théorie des fictions pourrait être utile aux sciences humaines. Pourquoi serait-il plus utile de parler de fictions pour réfléchir sur les divers travạux et objets des sociologues, historiens, psychologues, etc. que de toute autre notion? Le livre de Laval qui, de toute façon, donnera au lecteur qui ne connaît pas Bentham, de précieux renseignements sur son auvre, nous semble devoir être placé dans cette perspective ${ }^{17}$ et tirer toute sa force d'avoir esquissé un point de vue sur une théorie dynamique des formes culturelles.

En ce sens, nous voudrions suggérer très brièvement l'avantage que pourrait représenter une théorie de style benthamien par rapport à une théorie transcendantale et critique à l'égard des sciences, laquelle peut représenter aussi à l'heure actuelle, une heureuse tentative. Laval ne situe-t-il pas lui-même imprudemment Kant et Bentham comme étant du même côté ? Une des idées majeures de Bentham, bien soulignée par l'auteur du Pouvoir des fictions (p. 90 sqq.), est celle d'une quadruple démultiplication des idées (réelles et fictives) et des mots (dans leur acception réelle et fictive), donnant lieu à une quadruple dérive, car l'évolution de ces sens peut être différente. Dans Chrestomathia, Bentham montre bien toute les difficultés (en particulier, pédagogiques) qui résultent de la dérive différentielle des sens savants et des sens ordinaires. Mais cet instrument intéressant que nous livre ici Bentham pourrait avoir d'autres effets encore que suggère la longue réflexion de Laval ${ }^{18}$ sur l'exemple privilégié de l'espace; car il n'y a pas lieu d'enfermer le rapport des entités dans la seule forme du rapport des entités fictives aux entités réelles (du sens commun); il y a lieu au contraire de poursuivre ces rapports du côté des sciences elles-mêmes, entre des entités fictives. Ainsi, n'est-il pas frappant qu'une philosophie, qui se veut fondamentale et susceptible de rendre compte de toutes les autres sciences par le rapport critique qu'elle installe à leur égard, se fait de l'espace une idée que telle ou telle science qu'elle prétend fonder serait en mesure de contester? La théorie triviale de l'espace et du temps qui caractérise la philosophie transcendantale, au moins dans sa version kantienne, ne disqualifie-t-elle pas cette volonté de fondement à l'égard de sciences qui, comme la géométrie et la physique à partir du $\mathrm{xIx}^{\mathrm{e}}$ siècle, développeront des conceptions de l'espace et du temps autrement plus subtiles et plus complexes. Ainsi la distorsion du trivial et du sophistiqué se poursuit-elle à l'intérieur des sciences elles-mêmes, discréditant les prétentions transcen-

17. Laval, p. 81, voit, en effet, s'ouvrir avec Bentham « de riches perspectives sur le processus de connaissance comme maîtrise de la métaphorisation et pratique de la tromperie acceptable ». Il nous laisse toutefois, de ce point de vue, un peu sur notre faim.

18. Laval, p. 90, va jusqu'à dire, d'une formule aussi brillante qu'un peu imprudente, que "l'ontologie est une affaire d'espace". 
dantales. Il serait décidément imprudent de mettre Bentham du même côté que Kant...

Reste un dernier problème, qui concerne tous les auteurs anglais de l'âge classique et moderne, peut-être plus particulièrement Bentham, et qui, à lui seul, vaudrait bien un colloque : comment se fait-il que des doctrines si manifestement utiles aient été si soigneusement recouvertes, au point qu'on n'ait guère cherché à les traduire en français, et que, lorsqu'on ne les a pas purement et simplement méprisées, elles soient restées enfouies dans le fonds ancien de quelques grandes bibliothèques? Est-ce parce que l'utilité n'est jamais destinée qu'à développer des fantasmes d'utilité, fort puissants dans la critique, mais évanouissants dès lors qu'il s'agit pour eux de se poser?

Didier Nordon, Les Mathématiques pures n'existent pas. Éd. rev. et augm., dessins de Michel Mendìs France. Le Méjan, Actes Sud, 1993. $11,5 \times 21,8,178$ p., bibliogr. (Mathématiques et société).

Le livre est la réédition, révisée et augmentée d'un chapitre nouveau sur la preuve, de l'ouvrage paru en 1982. Il a gardé toute sa saveur et sa verve; il reste aussi suggestif qu'il y a dix ans; le philosophe y trouve partout, à lire ce texte écrit par un mathématicien bordelais, des esquisses d'analyses de concepts centraux, propres à solliciter sa réflexion sur les mathématiques, leur histoire et leur épistémologie; mais il y retrouve aussi les mêmes équivoques que par le passé.

Loin de nous l'idée de reprocher à Didier Nordon de ne pas donner de définition des mathématiques; les philosophes en ont produit de nombreuses dans le passé, toutes aussi imparfaites les unes que les autres ${ }^{19}$. Nul ne reprochera non plus à Nordon de sembler s'inspirer de l'esthétique moderne anglo-américaine pour parler du monde des mathématiciens et de ce qu'y font les mathématiciens en évitant de tomber dans le piège de définir de façon trop étroite l'objet et les méthodes des mathématiques. Mutatis mutandis, Nordon parle du « milieu » des mathématiciens à la façon dont Arthur Danto, Morris Weitz et George Dickie parlent du «monde de l'art »; et l'on découvre, non sans plaisir, que l'analogie mène assez loin. Si elle mène à une circularité ${ }^{20}$ et à des apories manifestes, elle n'en présente pas moins l'intérêt de fonder une recherche de nature quasi « ethnologique ". L'auteur montre comment une camaraderie de façade entre les

19. N'est-ce pas d'ailleurs le signe de la richesse d'une activité que l'on ne puisse en circonscrire exactement les limites?

20. P. 83 : «Définition $1:$ On appelle mathématiciens ceux qui font des mathématiques.

Définition 2 : On appelle mathématiques ce que font les mathématiciens. " 
mathématiciens voile la réalité d'une implacable hiérarchie, où les arguments d'autorité tiennent aussi souvent lieu de preuves que les démonstrations (p. 34-35, 72, 109-112); où la recherche est sclérosée par la loi d'airain du "publish or perish", tant dans son contenu que dans ses modes d'écriture qu'on voudrait prendre pour de la rigueur et qui consistent souvent en stéréotypes liés à la suprématie absolue de la langue anglaise. On trouvera, dans l'ouvrage, un savoureux sottisier de propos de mandarins, voire de mathématiciens plus légitimement prestigieux (p. 20-24, par ex.). Encore une fois, le caractère pamphlétaire de l'ouvrage, voire ses insolences, déclenchent le rire du lecteur, lequel comprend d'ailleurs que le monde des mathématiciens n'est pas le pire et qu'il conviendrait de soumettre à ce genre d'espièglerie d'autres mondes qui n'ont pas toujours trouvé, en dépit des grands airs d'authenticité qu'ils se donnent, la franchise d'un Nordon pour exhiber leurs travers, grands et petits.

Mais l'arme du pamphlet est dangereuse et se retourne aisément contre celui qui ne la manipule pas avec assez de précaution. La loi du genre porte à accorder une place considérable à ceux dont on conteste les positions et met à la portion congrue ceux qui détiennent les affirmations que l'on croit vraies, mais qu'on ne développe pas suffisamment : la suprématie donnée à l'attaque ne permet ni d'expliciter les positions que l'on tient, ni même de veiller suffisamment à leur cohérence. Deux exemples, particulièrement gênants, vont être donnés.

Après s'être servi de la thèse tout à fait légitime de Joseph Needham selon laquelle l'espace et le temps du marché moderne, qui tend à s'universaliser dès l'époque de la Renaissance, sont à l'origine d'une façon nouvelle de traiter l'espace et le temps en mathématiques, l'auteur cite une phrase d'Alexandre Koyré, laquelle, sortie de son contexte, dit que la science moderne a rejeté « toutes considérations basées sur les notions de valeur » (p. 37). Or, c'est tout le contraire que veut dire Nordon : si le lieu et l'instant n'ont plus, à l'âge classique, les valeurs que leur assignait le cosmos aristotélicien, ils en ont acquis d'autres « à l'étalon de valeur du marchand ", par exemple; mais, pour le montrer de façon convaincante, il faudrait l'établir dans le détail d'une démonstration mathématique, comme l'a fait avec rigueur Ernest Coumet en réfléchissant sur les principes du calcul des partis chez Cardan et Pascal. Il est vrai que des opérations qui apparaissent purement mathématiques ne sont pas mesurées que par le vrai et le faux, mais qu'elles sont pénétrées intimement, inextricablement, par une certaine idée de la justice des partages, des héritages, des entreprises. Il ne faut toutefois pas, en ce domaine, se contenter d'allusions pour convaincre, surtout quand celles-ci paraissent dire le contraire de ce qu'on veut montrer. 
Si riche le livre soit-il en exemples, il ne les développe pas toujours suffisamment pour échapper au reproche d'abstraction. Un autre exemple le montrera mieux encore. C'est un poncif de dire que la science occidentale - mathématiques comprises - participe, par sa volonté même d'être universelle et de valoir pour tout homme quel qu'il soit, du projet impérialiste de l'Occident (p. 27). Les conquêtes militaires et commerciales se sont appuyées sur les développements scientifiques pour réussir; on peut donc, en retour, porter ses soupçons sur l'activité scientifique elle-même et la faire apparaître comme un impérialisme inconscient, relevant du mécanisme idéologique bien connu de la «camera obscura ${ }^{21}$ ». Mais, à un pareil degré de généralité, qui donc, doué d'exigences scientifiques normales, peut se laisser convaincre par un tel argument? On sait depuis longtemps que l'une des armes favorites de celui qui veut tromper tient dans le recours aux idées générales, dont il exploite la multiplicité de sens ; Jeremy Bentham l'a parfaitement montré dans son Handbook of political fallacies. Pourquoi devrait-on faire confiance à la réfutation lorsqu'elle utilise les mêmes procédés que ceux qu'elle conteste? Tant qu'on ne montre pas de manière déterminée comment une science participe à l'asservissement d'autres hommes, on ne convainc guère et le remède, s'il n'induit pas quelque iatropathie, ne vaut à coup sûr guère mieux que le mal dont il est censé nous délivrer ${ }^{22}$.

Pourtant ces preuves d'avocat et autres pratiques fallacieuses ne doivent pas dissimuler le vrai problème posé par un livre dont le grand tort est de s'empresser de répondre à une question qui n'a pas été suffisamment précisée. On veut à tout prix nous convaincre, avec une ardeur militante, que " les mathématiques pures n'existent pas ", mais de quelle «pureté » s'agit-il? S'est-on seulement avisé de déterminer ce qu'on entendait par « pures » dans l'expression « mathématiques pures »? Le livre de Nordon souffre de l'équivoque qu'il laisse peser sur ce terme. Si le lecteur veut tirer quelque bénéfice du livre, il faut qu'il inspecte lui-même les divers

21. P. 28 : « Curieux paradoxe : au moment même où il se croit en dehors de toute idéologie, c'est-à-dire au moment où il proclame très haut le caractère universel de sa discipline et son essentielle indépendance vis-à-vis des circonstances sociales, économiques, culturelles, c'est à ce moment-là précisément que le mathématicien est en pleine idéologie. » Et peut-être plus encore celui qui la dénonce en utilisant les mêmes méthodes! " Ainsi se vont les opinions succédant du pour au contre, selon qu'on a de lumière $»$, comme le disait un fin dialecticien qui recherchait, dans ses « pensées », la « raison des effets ».

22. Notons au passage que cet usage malencontreux de la généralité n'empêche pas Didier NorDon, p. 12, de considérer la philosophie comme une sorte de science des idées générales et de le lui reprocher implicitement : « Tu vois la paille dans l'œil de ton prochain... »! Les rapports des mathématiciens et des philosophes sont décidément étranges : même quand ils ne sont pas empreints de mépris, ils donnent lieu à d'étranges méprises, comme celle de croire qu'ils sont " influencés par des systèmes de pensée » (p. 93), alors qu'ils le seraient plutôt moins que les autres. 
sens du mot «pur » en usage dans le texte et que, la plupart du temps, il restitue mentalement lui-même, à ses risques et périls, chacun de ces sens, quand il en a besoin. Essayons de le faire, car le propos du livre en vaut la peine, par la vivacité de son détail et par l'ensemble de son actuel chapitre $\mathrm{v}$, dont on pourrait aisément inverser les visées polémiques en pistes de recherches.

« Pur » est utilisé, tour à tour, dans les sens suivants, lesquels, confondus, compromettent la valeur des jugements qui usent du terme, à moins que le lecteur consente à faire de lui-même un effort que l'auteur n'a pas fait. D'abord, « pur » peut signifier « inné » et s'opposer aux artifices de construction $^{23}$; en second lieu, il peut vouloir dire « a priori » et s'opposer à ce qui dérive de l'expérience, qu'elle soit celle du monde physique ou du monde social; en troisième lieu, «pur » peut s'opposer à composé ${ }^{24}$; en quatrième lieu, « pur » qualifie des objets qui existent idéalement, séparément des actes de pensée que le mathématicien peut développer pour établir des propositions qui les concernent ${ }^{25}$; enfin, «pur » qualifie une activité prétendument indépendante de toute activité sociale, en particulier désintéressée de tout ce qui est économiquement utile ${ }^{26}$; ou, du moins, qui présente une certaine autonomie par rapport aux autres activités sociales ${ }^{27}$. Ces sens, s'ils se confirment parfois les uns par les autres, n'en doivent pas moins être soigneusement distingués. Car l'inné n'est pas l'a priori; une notion peut être a priori et être composée; non seulement l'a priori n'empêche pas l'activité mentale et la fabrication, mais il les requiert; de plus, la reconnaissance d'une activité sociale dans cette activité mentale ne saurait constituer une grande découverte, moins encore une découverte scandaleuse.

Faute de distinguer entre ces sens, l'auteur ne dit rien sur les points les plus authentiquement difficiles et du même coup, se contredit sur des points essentiels. Ainsi, le point difficile en ce qui concerne la pureté n'est pas de savoir si les mathématiques sont une activité sociale ou non, mais de savoir si l'on peut cerner ce qui est proprement mathématique dans une

23. Nordon, p. 161-162, oppose le « naturel » au « construit ». Ainsi, « l'idée qu'un objet a une permanence n'est pas une idée "naturelle", mais une idée construite ".

24. C'est ainsi que a l'intervention du temps en mathématiques était une de ces impuretés d'origine plus ou moins obscure donc incontrôlable (psychologique, qui sait, voire sociale?) dont il faut se méfier comme de l'irrationnel » (p. 156).

25. C'est la « croyance implicite " selon laquelle « il y a "quelque part" les mathématiques et, ici-bas, ses servants plus ou moins dignes d'elles » (p. 77).

26. Et qui, à ce titre, peut être belle. Voir p. 49. La neutralité politique peut aussi, en ce sens, faire partie de cette pureté (p. 59). «Si les mathématiques ont une richesse, c'est dans leur impureté qu'elle se trouve : pour le meilleur et pour le pire, les mathématiques font partie de la cité. $[\ldots]$ » (p. 137).

27. Ce que Nordon ne conteste nullement, voir p. $11:$ «On ne peut comprendre l'influence sociale des mathématiques en faisant totalement abstraction du sens des mathématiques ellesmêmes. " 
activité ou dans un objet que l'on qualifie ainsi. L'entrelacement des valeurs n'est-il pas tel qu'on ne puisse jamais isoler ni séparer ce qui est proprement mathématique? Et pourtant, les activités mathématiques, si hétérogènes soient-elles, se distinguent bien de celles qui ne le sont pas : à quel amalgame d'actes et de valeurs convient-il de donner ce nom de « mathématiques" À un certain moment, l'amalgame semble se fermer sur lui-même, prend une consistance propre qui fait que l'objet jouit d'une certaine autonomie, laquelle peut faire illusion. Le jeu des pensées sociales accumulées finit par se renverser en un être idéal qui paraît vivre d'une vie propre et se situer hors du temps (p. 149-150). C'est évidemment sur ce processus de renversement, source d'illusions, que nous souhaiterions voir porter les analyses : comment des processus mentaux hétéroclites se transforment-ils en objets ou en idéalités? Comment le langage intervient-il dans cette illusion d'idéalité et d'intemporalité ? Peut-être conviendrait-il de distinguer un cours (psychologique) des pensées d'un ordre des pensées (ou plutôt des idées, pour recourir à la distinction leibnizienne). Une théorie empiriste des mathématiques n'est nullement absurde; mais il faudrait parvenir à expliquer par ses genèses ce que donne, par ses descriptions, une conception «a prioriste ». Nordon nous lâche sur les points les plus précieux; son empirisme est de combat - d'attaque ou de défense; il ne devient jamais vraiment, sauf peut-être à de rares exceptions près ${ }^{28}$ une thèse.

Il est rare qu'une telle carence ne se solde pas par des contradictions. En voici au moins deux, particulièrement frappantes. On trouve la première lorsque Nordon écrit : «[...] les mathématiciens apportent un soin presque maniaque à définir parfaitement les objets qu'ils utilisent : même s'il leur arrive souvent d'être inconnus ou impossibles à expliciter, ces objets ont de droit une existence fixe et inaltérable, une identité » (p. 42). Ce reproche ne laisse pas d'être étonnant dès lors que l'on professe de ne pas croire à

28. Le chap. vі sur le rôle du temps en mathématiques est sans doute le plus riche sur ce thème ; mais il nous laisse sur notre faim lorsqu'il parle du * mystère * du " temps de la maturation psychologique » qui " imprègne toute démarche mathématique » (p. 152). On trouve toutefois, dans le même chapitre, une belle remarque sur Karl Weierstrass auquel « l'histoire des mathématiques » aurait « donné raison " - le temps est en principe éliminé de la théorie des fonctions - mais aussi auquel elle a " psychologiquement donné tort ": on emploie encore l'expression « $x$ tend vers a » avec une intuition de mouvement (p. 154). NoRDON, p. 41, retrouve cette distinction fondamentale en percevant mieux sa nécessité, lorsqu'il se livre à une «curieuse réflexion sur l'écriture des mathématiques, qui se lit à la fois de gauche à droite et de droite à gauche! Par exemple, afin d'effectuer le calcul de sin $x^{2}$, pour une valeur donnée $x$, il faut partir de cette valeur $x$ puis (lecture de gauche à droite) calculer $x^{2}$ et ensuite (lecture de droite à gauche) en prendre le sinus $»$. Il y a un cours psychologique des pensées et un ordre des idées, gagné et fabriqué à partir du précédent, mais qui a acquis cependant des caractères et surtout une temporalité spécifiques. 
l'existence séparée d'objets mathématiques ${ }^{29}$, dont la définition deviendrait alors une sorte de description. Si l'on construit l'objet mathématique, comment le mathématicien, pourrait-il se jouer la comédie que l'objet lui est inconnu? Plus exactement, il faudrait expliquer comment l'objet échappe à celui-là même qui le constitue par sa définition; ou, en termes leibniziens, comment se constituent, dans l'acte même de définir, des « idées sourdes ", c'est-à-dire qui restent partiellement inexploitées et inactives.

La seconde contradiction concerne l'une des questions sur lesquelles le livre se referme et s'apprête à conclure. Non pas qu'il soit scandaleux qu'un livre de polémique sur la rigueur et la pureté s'achève sur des questions. L'Analyste de Berkeley ne se ferme-t-il pas lui-même sur une série de superbes questions? Toutefois, ces questions, pour embarrassantes qu'elles soient, n'en sont pas moins parfaitement cohérentes avec le reste de l'ouvrage de l'évêque de Cloyne. Or on ne sait ce que l'on doit le plus incriminer chez notre mathématicien de Bordeaux, de l'inconséquence ou de la mauvaise foi, lorsque, après avoir passé son temps à nous convaincre que l'objet mathématique est une construction artificielle à partir d'actes composites que l'on décide de fixer en un objet, il semble reprocher au mathématicien de tenir un discours qui ignore la conscience de soi : « Peut-on parler vraiment sans parler de soi? » La question est belle, mais extravagante dans ce contexte, car, de deux choses l'une : ou l'on adopte une perspective "constructiviste" en mathématiques et l'on ne voit pas comment le discours mathématique pourrait être celui d'une connaissance ou d'une conscience de soi; ou l'on tient un discours innéiste, à la façon de Leibniz, et il n'est pas absurde de dire que nous nous connaissons nousmêmes par les mathématiques. Mais on ne peut pas demander une chose et son contraire, encore que les besoins de la polémique portent à passer, en un éclair, d'un point de vue au point de vue opposé.

Il manque à ce travail, brillant dans ses attaques, plus décevant dans ses affirmations et surtout dans leur cohérence, une philosophie qui permettrait de mieux les poser et de les relier plus systématiquement les unes aux autres. Il nous a semblé, en confrontant les textes, que Nordon pouvait trouver un devancier exigeant et tout à fait à la hauteur des problèmes qu'il pose en la personne de Jeremy Bentham qui, dans Chrestomathia, réfléchit sur les mathématiques de son temps, leur vérité, leur autorité, leur rigueur, leur utilité et leur enseignement; et que ce qui a manqué le plus à l'entreprise de Nordon, c'est une théorie des fictions qu'il avait pourtant tous les moyens de réactualiser. Il faut se pencher sur le chapitre $v$, qui reste

29. Il est vrai que l'on trouve une phrase assez énigmatique, p. 13, qui introduit le doute sur les véritables conceptions de l'auteur quant au statut construit ou donné des objets mathématiques. "Reproche-t-on à l'astronome de ne pas créer d'étoile? ", demande imprudemment l'auteur. 
le plus réussi de l'ouvrage; car le chapitre rajouté tend simplement à développer la remarque, déjà faite dans l'édition précédente que, dans la discipline réputée la plus rigoureuse, les progrès impliquent des phénomènes d'autorité.

Dans le chapitre v, l'auteur s'intéresse, sur des exemples particulièrement bien choisis et parfaitement convaincants, au rapport de la langue vernaculaire et de la langue des symboles utilisés en mathématiques. Si formelle que soit la langue du mathématicien, elle n'est pas sans relation avec la langue usuelle dans laquelle il faut bien désigner les objets et les opérations (p. 39-40), pour se comprendre soi-même, pour être compris des autres ${ }^{30}$ et pouvoir délivrer un enseignement ${ }^{31}$. C'est d'une façon très «benthamienne " que Nordon montre comment les sens rigoureux du terme de "fonction» ont dérivé à partir du sens usuel de ce terme (p. 135) ${ }^{32}$; tout se passe comme si les notions mathématiques étaient en rapport d'entités fictives à l'égard des entités réelles que sont les signifiés de la langue usuelle ${ }^{33}$. C'est encore dans le plus pur «benthamisme " que l'auteur montre que la dérive des termes usuels ne s'effectue pas au même rythme que celle des entités fictives forgées par le mathématicien; qu'il y a une temporalité différentielle de l'écoulement des uns et des autres. Il faut se garder de donner aux termes usuels par lesquels on se rapporte aux objets mathématiques le même sens à une époque et à une autre (p. 143) ${ }^{34}$; en ce sens, l'histoire des mathématiques peut jouer un rôle critique à l'égard des langues. L'accord avec Bentham va plus loin encore lorsque Nordon remarque que les différentes versions du « même » texte mathématique en anglais, en français, en russe ou en allemand n'ont pas la même valeur ${ }^{35}$. Incorporé dans des systèmes de références différents, le texte n'a

30. Encore qu'on puisse faire des mathématiques sans les comprendre de la même façon (p. 145).

31. Comme chez Bentham, le dernier mot de Nordon revient toujours à la pédagogie. "L'expression "comprendre les mathématiques" n'est guère moins mystérieuse quand il s'agit d'une conversation mathématique que quand il s'agit de n'importe quel type de conversation " (p. 145). Le chap. v, p. 146, se termine sur cette belle formule : " [...] la pédagogie des mathématiques est un problème difficile que la perfection du discours mathématique ne résout pas."

32. Le mot « indépendant » en calcul des probabilités donne lieu à des remarques comparables (p. 138, 146).

33. P. 135-136: «Sens usuel et sens mathématique agissent et réagissent constamment l'un sur l'autre; les mathématiciens se sont servis du sens usuel, se sont appuyés sur lui, en ont eu besoin. »

34. NORDon, p. 143-144, montre cette idée avec finesse sur l'exemple privilégié de la notion de nombre.

35. Chaque langue porte avec elle un imaginaire. Même entre des langues très proches comme le français et l'anglais, la correspondance est loin de s'effectuer facilement : si le terme mathématique d' " anneau " correspond bien à l'anglais « ring ", celui de "corps" se traduit par « field » en anglais (p. 140-141). Sur l'exemple de la distinction cantorienne des " reellen Zahlen " et des " realen Zahlen ", NoRDoN, p. 128, montre comment une difficulté de traduction peut se solder par l'élimination d'une idée. 
pas d'identité aisément repérable; l'universalité du sens d'un texte n'est jamais que visée à l'entrecroisement des divers points de vue que représentent les diverses langues, mais elle ne saurait être à jamais saisie en une seule. La traduction d'un texte mathématique d'une langue dans une autre est un véritable changement de signification (p. 40). Et l'on comprend alors que la suprématie de la langue anglaise sur toutes les autres pour la rédaction de textes mathématiques n'est pas sans conséquence pour les mathématiques elles-mêmes ${ }^{36}$.

Pour toutes ces raisons - et pour beaucoup d'autres encore ${ }^{37}$, à commencer par un utilitarisme critique, mais insistant, affiché par l'auteur (p. 50, 53, 66-69, 96, 104, 107-108) —, Nordon est benthamien, peut-être comme monsieur Jourdain faisait de la prose. Mais c'est moins ce rattachement au passé qui nous intéresse que la réouverture de perspectives très semblables à celles de Chrestomathia par-delà l'évolution que les mathématiques ont pu prendre en s'engageant dans les voies du formalisme. On trouve chez Nordon cette belle remarque en commentaire du fameux texte dans lequel David Hilbert prétend qu'il aurait pu remplacer « point, droite, plan » par «table, chaise, bock de bière » et, par conséquent, pu écrire que « par deux bocks de bière passe une table et une seule »: " Il ne l'a pas fait $^{38}$." Cette remarque est précieuse et montre bien que le formalisme reste toujours une « formalisation » et n'est qu'une visée idéale et séparatiste, projection d'une activité "qui consiste à se défaire », par degrés, comme on multiplie les ordres de fictions à partir des entités réelles ${ }^{39}$, " de

36. C'était l'hypothèse de Benjamin Lee Whorf à laquelle se réfère explicitement NoRDoN, p. 140 : « L'approche de la réalité à laquelle la science moderne est en train de se livrer par le biais des mathématiques n'est qu'une approche à travers un cas particulier de la relation existant entre la réalité et le langage. »

37. Curieusement, la discrète référence à Lacan (p. 126) pourrait bien servir aussi de lien à Bentham. Lacan a sans doute été l'un des premiers en France à s'intéresser à la théorie des fictions, qui avait fait l'objet du recueil d'OGDEN, voir op. cit. supra n. 2. On sait qu'HALEvY, in op. cit. supra n. 1, avait malencontreusement méprisé cette partie de la recherche de Bentham.

38. Et pas davantage Nicolas Bourbaki, lequel « a toujours été guidé dans son choix des mots par leur sens "intuitif" et "naturel" " (p. 132).

39. On voit très bien ces ordres de fictions se constituer dans cette belle analyse de NorDoN, p. 131 : « Lorsque J.-L. Krivine écrit : "Cette définition des mots "fini" et "entier naturel" leur donne un sens tout à fait différent du sens habituel », l'expression "sens habituel" désigne pour lui le sens habituel des mathématiciens. Et comme ce sens est à son tour sensiblement distinct du sens de l'"homme de la rue" (en particulier en ce qui concerne le mot "fini"), nous voici en présence, pour un mot donné, de divers sens - le sens logique, le sens mathématique, les sens usuels - intimement liés entre eux, dont chacun nourrit les autres, mais pourtant bien distincts. " Un peu auparavant, l'auteur avait remarqué, p. 125, que « la pertinence de l'allusion du sens mathématique au sens usuel [pouvait] varier d'un mot à l'autre » et y avait vu l'indice que les objets mathématiques «n'ont pas tous le même statut ni le même rapport à la réalité ». La discussion sur le terme " réels » appliqué aux « nombres " est également d'une très grande finesse. NorDoN, p. 128, montre en particulier comment le développement de l'informatique a fait évoluer la notion de "réalité » d'un nombre : « Le critère de "réalité" d'un nombre est qu'il puisse apparaître sur un écran. " 
la prégnance du sens courant » (p. 123). Nordon n'a pas son pareil pour scruter avec finesse les hésitations des mathématiciens dans leurs désignations, sonder les choix inconscients ${ }^{40}$ qui président à la sélection des termes, repérer les difficultés qu'ils rencontrent à accorder tel terme avec tel autre ${ }^{41}$; mais aussi les raisons secrètes de l'engouement de tel ou tel public, plus ou moins savant, pour les «ensembles» (p. 45-47), les « groupes» (p. 138) ou la « théorie des catastrophes » (p. 70, 138-139).

Par le pétillement de ses exemples et le fourmillement de ses remarques critiques, le livre de Nordon appelle un livre plus général qui analyserait de près le double usage du langage et construirait la théorie de cette double association de catégories différentes de signes. Il n'est pas impossible qu'une telle théorie nous mènerait à mieux envisager les rapports entre les remarques sur le langage et celles qui concernent le temps en mathématiques. Le chapitre vi sur le temps est suggestif, quoique un peu confus, puisque l'auteur semble reprocher aux mathématiques de ne pas incorporer dans leur recherche leur propre histoire (p. 155, 162) et de ne pas être un discours dialectique. De plus, il ne revient peut-être pas tout à fait au même d'accorder une temporalité à certains objets mathématiques et d'en accorder une au cours même de la démonstration ${ }^{42}$. En revanche, par le biais des réflexions sur le travail inégal des noms et des verbes dans les textes mathématiques $^{43}$, il y aurait peut-être matière, si l'on se rappelle que le verbe est l'opérateur du temps dans le discours, à une unification des remarques des chapitres $v$ et vI.

Par ses coups de sonde épars, le livre de Nordon peut donc être considéré comme l'ébauche d'un ouvrage plus général sur l'ontologie des objets et des actes du mathématicien; il serait difficile de lui en faire le reproche, d'autant que l'ontologie a plus coutume de se faire désirer que de se réaliser. Cette ontologie peut être envisagée comme une région comprise dans

40. La référence à $M$. Nguyen Thanh Liem est, de ce point de vue, intéressante.

41. Faut-il mettre un « $S$ » à « standard », lorsqu'on parle d'objets standard(s)? Faut-il parler de « fonctions standardes »?

42. Tous ces points nous ramènent à la distinction du cours du temps et de l'ordre du temps, qui est, comme on sait, au coeur de la philosophie transcendantale, mais dont une philosophie empirique doit certainement pouvoir rendre compte.

43. P. 141-142: «Quand les mathématiciens réutilisent un mot usuel en lui donnant un sens mathématique, ils le font presque toujours pour un nom, un adjectif ou un adverbe et presque jamais pour un verbe ("opérer sur", "tendre vers", "projeter" sont quelques rares exceptions) et, bien entendu, jamais la fonction grammaticale d'un mot ou d'une idée ne change entre l'usage mathématique et l'usage habituel : l'ensemble des verbes et le système d'oppositions verbes/noms offerts par les langues européennes conviennent aux besoins des mathématiciens. " La lecture des traités de probabilité des Xvir et xvir siècles permettrait de nuancer la prétendue pauvreté de l'usage des verbes par le mathématicien. Il est toutefois indiscutable que, même dans ces traités où elles sont plus riches que dans les autres, la syntaxe et la grammaire des modes et des temps des verbes ne reçoivent aucun changement. 
le vaste territoire d'une théorie générale des fictions. Ces recherches, quand elles n'ont pas été purement et simplement écartées ${ }^{44}$, ont été trop tôt interrompues. Les cuvres de Bentham sont, sur ce point, dans un état de chantier. Il faudrait, après un inventaire de ce chantier, reprendre un certain nombre de fondations et tenter de rebâtir à nouveaux frais.

Ainsi, ce qui est frappant chez Nordon, c'est la reprise - et même l'insistance dans la reprise par un livre qui méritait pleinement sa seconde édition - de thèmes assez mal connus des Français, mais qui trouvent des développements déjà importants, deux siècles auparavant, chez Bentham. Nordon note que le jeu des sens des mots usuels et des symboles est celui d'une modification réciproque ${ }^{45}$; Bentham, dans Chrestomathia, montrait que la théorie des fictions permettait de rendre compte du développement des mathématiques, de leur enseignement, et que, en même temps, elle était transformée par ce dont elle était censée rendre compte. On trouve chez Nordon l'exploitation de thèmes que l'on aurait pu croire épuisés au début du $\mathrm{xIx}^{\mathrm{e}}$ siècle et incapables de concerner l'homme du $\mathrm{xx}^{\mathrm{e}}$ qui a connu, sur le terrain des mathématiques, depuis cette époque, de prodigieux changements. Par la qualité de ses exemples et de ses suggestions, Nordon donne le désir de faire, pour notre époque, une théorie moderne des fictions. Il est remarquable que son texte ne se réfère pas une seule fois à Gaston Bachelard, comme si la perspective des "obstacles épistémologiques", quoiqu'elle fût solidaire d'une théorie psychanalytique, ne lui servait à rien. Vraisemblablement, la double raison en est, d'une part, dans l'intérêt primordial accordé par l'auteur aux mathématiques ${ }^{46}$, plutôt qu'à la phy-

44. Halévy, pourtant si soigneux dans ses études sur Bentham, écarte sans ménagement les travaux proliférants de Bentham dans ce domaine de l'ontologie. Or on pourrait se demander si elles ne constituent pas l'essentiel de l'cuvre et comme le fondement de toutes les analyses : économiques, politiques, juridiques.

45. P. 154 : * Employer un mot, c'est accepter son sens, mais c'est aussi contribuer à en construire le sens.

46. On trouve chez Nordon la juste intuition que c'est dans le pouvoir de transcendance des mots que tient une bonne partie des illusions des mathématiques. Voir, p. 124-125: * Les mathématiques n'ont pas la réalité physique comme référent ultime et unique. Leurs objets sont pris dans la langue. Le travail du mathématicien a un aspect "littéraire". N'hésitons pas à le dire : Bourbaki fut un véritable écrivain [...] Abusés par les mots simples et concrets qu'ils employaient, [les bourbakistes] ont fini par prendre pour "réelles" des élaborations qui n'étaient somme toute "que bien écrites" [...]. " Cette intuition rattache plus notre auteur à la tradition anglo-saxonne qu'à une conception bachelardienne des * obstacles ". 
sique ou à quelque science expérimentale, d'autre part, dans les convictions empiristes de Nordon, lesquelles se trouveront mieux servies par une théorie des fictions.

Jean-Pierre CĹéro

Faculté des lettres et sciences humaines

Université de Rouen

76821 Mont-Saint-Aignan Cedex

(septembre 1994). 